Tropical Journal of Pharmaceutical Research September 2015; 14 (9): 1549-1556

ISSN: $1596-5996$ (print); 1596-9827 (electronic)

(C) Pharmacotherapy Group, Faculty of Pharmacy, University of Benin, Benin City, 300001 Nigeria.

All rights reserved.

Available online at http://www.tjpr.org

Original Research Article

http://dx.doi.org/10.4314/tjpr.v14i9.2

\title{
Preparation, Characterization and Evaluation of Elvitegravir-Loaded Solid Lipid Nanoparticles for Enhanced Solubility and Dissolution Rate
}

\author{
Pavan Kommavarapu*, Arthanareeswari Maruthapillai and Kamaraj Palanisamy \\ Department of Chemistry, SRM University, Kattankulathur - 603203, Kancheepuram District, Tamilnadu, India
}

*For correspondence: Email: kommavarapu.pavan@gmail.com; Tel: +918106791313

Received: 6 March 2015

Revised accepted: 29 July 2015

\begin{abstract}
Purpose: To enhance the aqueous solubility and dissolution rate of elvitegravir (EVG) by formulating the drug as solid lipid nanoparticles (SLNs) using solvent injection method.

Methods: EVG-loaded SLNs were prepared by solvent injection method. Four different formulations of SLN were prepared using gelucire - 44/14 as lipid core in ethanol, soya lecithin as emulsifier, and polysorbate 80 as surfactant in the aqueous phase. The SLNs were characterized for various physical properties, including particle size, zeta potential, polydispersity, release profile and entrapment efficiency.

Results: The yield of SLNs was in the range $151.0 \pm 2.4$ to $199.1 \pm 2.7 \mathrm{~nm}$. Significant changes were observed in mean particle size ( $\mathrm{nm}), Z$ - potential $(\mathrm{mV})$ and polydispersity index (PDI) of the SLNs by varying the concentration of cryoprotectant. EVG - SLNs demonstrated a 800 - 1030-fold enhancement in aqueous solubility compared with plain EVG. The dissolution efficiency (DE) for SLNs was > $63 \%$ in all cases and increased up to $83 \%$ with increasing lipid load.

Conclusion: Successful preparation and characterization of elvitegravir-loaded solid lipid nanoparticles by solvent injection method has been accomplished in this study.
\end{abstract}

Keywords: Elvitegravir, Solid lipid nanoparticles, Cryoprotectant, Lipid load, Entrapment efficiency

Tropical Journal of Pharmaceutical Research is indexed by Science Citation Index (SciSearch), Scopus, International Pharmaceutical Abstract, Chemical Abstracts, Embase, Index Copernicus, EBSCO, African Index Medicus, JournalSeek, Journal Citation Reports/Science Edition, Directory of Open Access Journals (DOAJ), African Journal Online, Bioline International, Open-J-Gate and Pharmacy Abstracts

\section{INTRODUCTION}

Solid lipid nanoparticles are colloidal carrier systems for controlled drug release. The main advantage of SLN's over liposomes, polymeric nanoparticles and polyester nanoparticles is the lipid matrix which decreases the danger of acute and chronic toxicity and has good tolerability [13]. The other important application of SLN's is improved bioavailability by oral administration and increased targeting ability of the drug $[4,5]$.

Various methods are available for the preparation SLN'S which include high pressure homogenization, high speed homogenization, micro emulsion based SLN preparation, double emulsion method, solvent emulsification/ evaporation, spray drying method, super critical fluid method and solvent injection method [1,6-8]. The present investigation uses solvent injection method to prepare EVG-loaded SLNs which is based on the injection of organic phase containing drug in to continuously stirred aqueous phase containing emulsifier and surfactant [9-11].

Elvitegravir is a low molecular mass drug $(447.883 \mathrm{~g} / \mathrm{mol})$ with the chemical name, $6-[(3-$ Chloro-2-fluorophenyl)methyl]-1-[(2S)-1-hydroxy- 
3-methylbutan-2-yl]-7-methoxy-4-oxoquinoline-3carboxylic acid, and empirical formula, $\mathrm{C}_{23} \mathrm{H}_{23} \mathrm{CIFNO}_{5}[12,13]$. The chemical structure of $E V G$ is shown in Figure 1.<smiles>COc1cc2c(cc1Cc1cccc(Cl)c1F)c(=O)c(C(=O)O)cn2[C@@H](CO)C(C)C</smiles>

Figure 1: Chemical structure of Elvitegravir

EVG belongs to a class of HIV drugs called integrase inhibitors which blocks HIV enzyme called integrase [14]. EVG is characterised with poor aqueous solubility so the main intend of the present study is to prepare EVG-loaded SLNs by solvent injection method with enhanced aqueous solubility and dissolution rate.

\section{EXPERIMENTAL}

EVG was supplied by PharmaTrain (Hyderabad, India), Gelucire 44/14 was a gift sample from Gattefossé (France), Ethanol Absolute $99.9 \%$ was from Commercial Alcohols (Brampton, Canada), Soya lecithin was from VWR International Ltd (Poole, England), polysorbate 20 was from Merck Specialities Pvt Ltd (Worli, Mumbai), Ultra-pure water (Millipore®, USA) was used for the preparation of aqueous phase. Dmannitol ( $\geq 98 \%$ ) here which is used as a cryoprotectant was from Sigma-Aldrich (Saint Louis, USA). All the chemicals used were analytical reagent grade and used without further purification.

\section{Preparation of EVG-SLNs}

The EVG-loaded SLNs (EVG-SLNs) were prepared by solvent injection method followed ultra-sonic homogenization. In brief, 300 mg EVG and $150 \mathrm{mg}$ Gelucire 44/14 were dissolved in ethanol which constitutes organic phase. The aqueous phase consists of $1 \% \mathrm{w} / \mathrm{v}$ polysorbate 20 as a surfactant and $1 \% \mathrm{w} / \mathrm{v}$ soya lecithin as an emulsifier in water. The organic phase $(5 \mathrm{ml})$ was injected rapidly through an injection needle in to continuously stirred aqueous phase (45 ml). The precipitate was stirred at $1500 \mathrm{rpm}$ for 120 min. The resultant SLN suspension (EVG SLN1) was homogenised in an Ultrasonic Homogenizer for 30 min. Similarly, three more
SLN suspensions EVG - SLN2, EVG - SLN3 and EVG -SLN4 were prepared by varying lipid weight $300 \mathrm{mg}, 450 \mathrm{mg}$ and $600 \mathrm{mg}$ respectively. EVG - SLNs were optimized for lipid concentration and the effect of variation of lipid ratio on particle size, zeta potential, polydispersity index, entrapment efficiency and drug release kinetics were evaluated. Effect of addition of cryo-protectant on particle size, zeta potential and PI were studied up on immersing EVG - SLN's in to liquid nitrogen.

\section{Particle size analysis}

The mean particle size ( $Z$ - Average) analysis and polydispersity index (PDI) were determined by dynamic light scattering (DLS) principle (Zetasizer nano ZS, Malvern Instruments, UK) after appropriate dilutions in Milli $Q$ water filtered through $0.22 \mu \mathrm{m}$ polyvinylidene difluoride (PVDF) filters. Size measurement was carried out in triplicate following proper dilutions in Milli $Q$ water at $25^{\circ} \mathrm{C}$ using DTS0012 -clear disposable sizing cuvettes with equilibration time $120 \mathrm{~s}$. The test was conducted at an angle of $173^{\circ}$ backscatter (NBS default) with measurement position set at $4.65 \mathrm{~mm}$.

\section{Zeta potential analysis}

The electrophoretic mobility of nanoparticles was determined using Electrophoretic Light Scattering principle (zetasizer nano ZS, Malvern Instruments, UK). Measurements were carried out in triplicate at $25{ }^{\circ} \mathrm{C}$ using water as a dispersant (refractive index: 1.330) in a clear disposable zeta cell.

\section{Morphological characterization by SEM measurement}

The morphology of the nanoparticles was investigated by scanning electron microscopy (SEM, JOEL JSM-6380) at an accelerating voltage of $10 \mathrm{kV}$. Double coated carbon conductive tabs were mounted on SEM sample stubs and pasted round glass coverslips on it and the sample was transferred and spread uniformly on the coverslips and conductive carbon paint is applied along the edges of coverslips and the sample is allowed to air dry. The samples were coated by Platinum Sputter Coater vacuum coater (JEOL, JFC 1600, Auto fine Coater) to minimize electrostatic charging.

\section{Solubility measurement of EVG and EVG- SLNs}

The equilibrium solubility of the pure drug EVG in water was determined by the traditional shake 
flask method. According to this method the drug is added in surplus to $5 \mathrm{ml}$ of water and shaken for $24 \mathrm{~h}$ at $37^{\circ} \mathrm{C}$ until equilibrium is reached. The saturation is confirmed by observation of the presence of un-dissolved material. After separation of the solid by filtration, the concentration of the drug in the filtrate was determined by UV Vis Spectrophotometer (LABINDIA-UV $3000+$ ) at $257 \mathrm{~nm}$ against known standard concentration preparation. For the estimation of soluble fraction of drug in EVG SLN's, $5 \mathrm{ml}$ of the suspension is taken and filtered through $0.45 \mu \mathrm{m}$ filters and determined the solubility of EVG in EVG - SLN's against known standard at $257 \mathrm{~nm}$.

\section{Determination of drug entrapment efficiency}

EVG-SLN's were centrifuged at $15000 \mathrm{rpm}$ at 5 ${ }^{\circ} \mathrm{C}$ for 30 min using Thermo Scientific IEC CL31 Multispeed centrifuge. Entrapment of EVG in EVG-SLN's was determined as in Eq 1.

Entrapment $(\%)=\{(\mathrm{W} 1-\mathrm{W} 2) / \mathrm{W} 1\} 100$

where $\mathrm{W} 1$ is the total amount of drug added during EVG-SLN's preparations. W2 is the amount of drug present in the clear supernatant layer, determined by UV-Vis spectrophotometer at $257 \mathrm{~nm}$.

\section{In vitro dissolution studies}

In vitro release of the drug and EVG SLN's was studied in USP-II apparatus using $2 \%$ polysorbate 80 in $0.01 \mathrm{~N}$ Hydrochloric acid as dissolution medium. The medium was stirred at $75 \mathrm{rpm}$ at $37^{\circ} \mathrm{C} \pm 0.5^{\circ} \mathrm{C}$. EVG SLN's suspension equivalent to $5 \mathrm{mg}$ of $\mathrm{EVG}$ was transferred into dissolution medium. At predetermined time intervals (ranging from 10 to $60 \mathrm{~min}$ ), $5 \mathrm{ml}$ of the sample medium was taken and the same amount of fresh medium was replaced. The amount of EVG released was determined using its absorbance at $257 \mathrm{~nm}$. Different mathematical models were applied to the dissolution data of EVG - SLN's to study the drug release kinetics. The most common mathematical models used for the evaluation of dissolution profile are listed in Table 1. Where Qt amount (\%) of drug substance released at the time t, Q0 is the start value of $\mathrm{Qt}, \mathrm{t}$ is the time, $\mathrm{n}$ is the release exponent, $a$ is the scale parameter, $b$ is the shape parameter and $\mathrm{K} 0, \mathrm{~K} 1, \mathrm{~K}, \mathrm{KS}$ and $\mathrm{KH}$ are the constants corresponding to respective models.

\section{Statistical analysis}

Data were analysed by Microsoft Office Enterprise 2007, Version 12.0.6612.1000. Statistical assessment of dissolution data of EVG and EVG SLNs was done by ANOVA: single factor and t-test; paired two-sample for means, and differences were set $p<0.05$.

\section{RESULTS}

Solvent injection method was selected for the preparation of EVG loaded SLN's which depends on the rapid diffusion of solvent across the solvent lipid interface with the aqueous phase [15]. EVG - SLN's were optimized for process variables like stirring speed, stirring time, sonication time and the effect of variation in lipid concentration on particle size, PDI and EE. The mean particle size of EVG - SLN1, EVG - SLN2, EVG - SLN3 and EVG - SLN4 were found to be 151.0, 174.8, 182.3 and $199.1 \mathrm{~nm}$, respectively, with unimodal distribution and PDI value is $<0.5$ throughout the study. The results are tabulated in Table 2.

Table 1: Kinetic models for dissolution profile evaluation

\begin{tabular}{ll}
\hline Kinetic model & Mathematical equation \\
\hline Zero order kinetic model & $Q_{t}=Q_{0}+K_{0} t$ \\
First order kinetic model & $\ln Q_{t}=\ln Q_{0}+K_{1} t$ \\
Second order kinetic model & $1 / Q_{t}=K t+1 / Q_{0}$ \\
Third order kinetic model & $1 / Q_{t}{ }^{2}=K t+1 / Q_{0}{ }^{2}$ \\
Korsmeyer-Peppas model & $Q_{t}=k t^{n}$ \\
Weibull model & $\left.\log ^{\mathrm{n}}-\ln \left(1-\left(Q_{t} / Q_{\infty}\right)\right)\right]=b X \log t-\log a$ \\
Hixson-Crowell model & $Q_{0}{ }^{1 / 3}-Q_{t}{ }^{1 / 3}=K_{S} t$ \\
Higuchi model & $Q_{t}=K_{H} \sqrt{t}$ \\
\hline
\end{tabular}


Table 2: Mean particle size $(\mathrm{nm}), \mathrm{Z}$ - potential $(\mathrm{mV})$, polydispersity index (PDI) and entrapment efficiency (\%) of EVG-SLN's containing different percentages of Gelucire 44/14 $(n=3)$

\begin{tabular}{lcccc}
\hline Formulation & $\begin{array}{c}\text { Mean particle size } \\
(\mathbf{n m})\end{array}$ & $\begin{array}{c}\text { Z-potential } \\
(\mathbf{m V})\end{array}$ & $\begin{array}{c}\text { Polydispersity index } \\
(\text { PDI) }\end{array}$ & $\begin{array}{c}\text { Entrapment efficiency } \\
(\%)\end{array}$ \\
\hline EVG - SLN1 & $151.0 \pm 2.4$ & $-41.4 \pm 1.6$ & $0.314 \pm 0.051$ & $77.3 \pm 1.56$ \\
EVG - SLN2 & $174.8 \pm 3.2$ & $-38.9 \pm 0.4$ & $0.383 \pm 0.032$ & $80.8 \pm 0.86$ \\
EVG - SLN3 & $182.3 \pm 1.8$ & $-39.8 \pm 0.7$ & $0.408 \pm 0.036$ & $84.1 \pm 1.81$ \\
EVG - SLN4 & $199.1 \pm 2.7$ & $-37.4 \pm 1.0$ & $0.420 \pm 0.029$ & $89.7 \pm 0.27$ \\
\hline
\end{tabular}

As the amount of lipid concentration increased the particle size of EVG - SLN's increased from $151.0 \mathrm{~nm}$ to $199.1 \mathrm{~nm}$. Table 2 also reports the $Z$ - potential values of EVG - SLN indicating the formation of stable suspension. The entrapment efficiency (\%) of EVG-SLN's was greater than 75 $\%$ for all the preparations and increased as the lipid load increase.

Initially, when the stirring rate was for $30 \mathrm{~min}$ uneven aggregates were observed resulting in non-homogenous suspension. The irregular aggregates with higher particles and high PDI value is due to increased viscosity of organic phase because of increasing lipid concentration resulting in slower diffusion of organic phase in the outer phase. Further increasing the stirring time from $30 \mathrm{~min}$ to $120 \mathrm{~min}$ resulted in homogenous suspension with low particle size and low PDI values. When the ultra sonic homogenization increased from $15 \mathrm{~min}$ to $30 \mathrm{~min}$ resulted in uniform particles with less particle size diameter. On further increasing the homogenization time to $45 \mathrm{~min}$, no significant change in particle size is observed.

Lyophilization of particles was carried out using mannitol to limit the risk of aggregation of particles. EVG - SLN nanosuspension's containing 2, 5, 10 and $15 \%$ mannitol as cryoprotectant was snap-frozen in liquid nitrogen. EVG - SLN suspensions without the addition of cryoprotectant were immersed in liquid nitrogen and found that mean particle size for EVG SLN1, EVG - SLN2, EVG - SLN3 and EVG SLN4 were 124.8, 141.9, 139.3 and $165.1 \mathrm{~nm}$ respectively. It is found that the mean particle size is decreased when compared to initial values and found that the $Z$ - potential values ranged from -17.2 to $-5.5 \mathrm{mV}$ indicating the decrease in stability of the EVG - SLN's up on immersing in liquid nitrogen. Effect of addition of different concentrations of mannitol as cryoprotectant on mean particle size, Z potential and PDI are studied and the results are tabulated in Table 3.
From these results it is found that for all the EVG - SLN preparations as the cryoprotectant concentration increased mean particle size ranging between $155.4 \mathrm{~nm}$ to $648.9 \mathrm{~nm}$. The $Z$ potential value for EVG - SLN1 and EVG-SLN2 ranged from - $47.4 \mathrm{mV}$ to $-32.1 \mathrm{mV}$ indicating the stability up on snap freezing but for EVG SLN3 and EVG - SLN4 the Z - potential value decreased significantly and the values ranged from $-29.5 \mathrm{mV}$ to $-8.6 \mathrm{mV}$ indicating the reduced stability which may be due to increased particle aggregation. The PDI value is less than 0.5 for EVG - SLN1 and EVG - SLN2 with narrow particle size distribution indicating polydisperse colloidal dispersion and greater than 0.5 for EVG - SLN3 and increased further for EVG - SLN4.

Imaging of SLNs by SEM is expected to provide information on SLN morphology and size. SEM images of the SLNs derived EVG are presented in Figure 2. Micrographs of SEM confirm that the EVG - SLN's are circular in shape.

The solubility results show that the aqueous solubility of EVG is $0.0016 \mathrm{mg} / \mathrm{ml}$ which indicates the practically insoluble nature of the drug. After the formation of SLN's the solubility of EVG SLN1, EVG - SLN2, EVG - SLN3 and EVG SLN4 increased to $1.29,1.47,1.57$ and 1.65 $\mathrm{mg} / \mathrm{ml}$, respectively, an increase in solubility ranging from 806 to 1031 -fold, respectively.

\section{In vitro dissolution}

EVG exhibits slow dissolution rate, only about 16 $\%$ of the drug was dissolved in first $15 \mathrm{~min}$ and about $40 \%$ of the drug was dissolved after 60 min. After SLN formation in all the cases drug release was more than $50 \%$ and increased up to $74 \%$ as the lipid load increased. The drug release profiles from the EVG-SLN's prepared by the solvent injection method in aqueous system exhibited a linear relationship for Weibull model after the burst of drug (Figure 3). 
Table 3: Effect of various concentrations of cryoprotectant on mean particle size $(\mathrm{nm}), \mathrm{Z}$ - potential $(\mathrm{mV})$ and polydispersity index (PDI) of the EVG - SLN'S $(n=3)$

\begin{tabular}{|c|c|c|c|c|}
\hline Formulation & $\begin{array}{c}\text { Mannitol } \\
\text { concentration }\end{array}$ & $\begin{array}{c}\text { Mean particle size } \\
(\mathrm{nm})\end{array}$ & $\begin{array}{c}\text { Z-potential } \\
(\mathrm{mV})\end{array}$ & $\begin{array}{c}\text { Polydispersity index } \\
\text { (PDI) }\end{array}$ \\
\hline \multirow[t]{4}{*}{ EVG-SLN1 } & $2 \%$ & $155.4 \pm 2.8$ & $-44.7 \pm 2.1$ & $0.380 \pm 0.016$ \\
\hline & $5 \%$ & $212.3 \pm 3.3$ & $-46.4 \pm 0.9$ & $0.321 \pm 0.011$ \\
\hline & $10 \%$ & $345.9 \pm 2.3$ & $-41.3 \pm 1.7$ & $0.397 \pm 0.014$ \\
\hline & $15 \%$ & $453.8 \pm 1.8$ & $-35.6 \pm 2.2$ & $0.388 \pm 0.009$ \\
\hline \multirow[t]{4}{*}{ EVG-SLN2 } & $2 \%$ & $181.1 \pm 2.5$ & $-43.8 \pm 3.2$ & $0.435 \pm 0.012$ \\
\hline & $5 \%$ & $314.6 \pm 5.8$ & $-45.2 \pm 2.1$ & $0.311 \pm 0.012$ \\
\hline & $10 \%$ & $428.1 \pm 4.4$ & $-47.4 \pm 4.3$ & $0.287 \pm 0.023$ \\
\hline & $15 \%$ & $583.3 \pm 3.2$ & $-32.1 \pm 1.1$ & $0.398 \pm 0.013$ \\
\hline \multirow[t]{4}{*}{ EVG-SLN3 } & $2 \%$ & $190.6 \pm 3.8$ & $-29.5 \pm 0.7$ & $0.545 \pm 0.019$ \\
\hline & $5 \%$ & $321.0 \pm 5.2$ & $-22.8 \pm 1.8$ & $0.593 \pm 0.018$ \\
\hline & $10 \%$ & $500.2 \pm 3.1$ & $-24.9 \pm 4.1$ & $0.653 \pm 0.011$ \\
\hline & $15 \%$ & $612.2 \pm 2.7$ & $-22.2 \pm 3.3$ & $0.698 \pm 0.020$ \\
\hline \multirow[t]{4}{*}{ EVG-SLN4 } & $2 \%$ & $210.6 \pm 1.9$ & $-17.8 \pm 4.2$ & $0.726 \pm 0.012$ \\
\hline & $5 \%$ & $398.4 \pm 3.0$ & $-13.3 \pm 0.9$ & $0.965 \pm 0.020$ \\
\hline & $10 \%$ & $498.9 \pm 2.2$ & $-13.1 \pm 3.8$ & $1.075 \pm 0.011$ \\
\hline & $15 \%$ & $648.9 \pm 4.7$ & $-8.60 \pm 3.6$ & $1.132 \pm 0.018$ \\
\hline
\end{tabular}
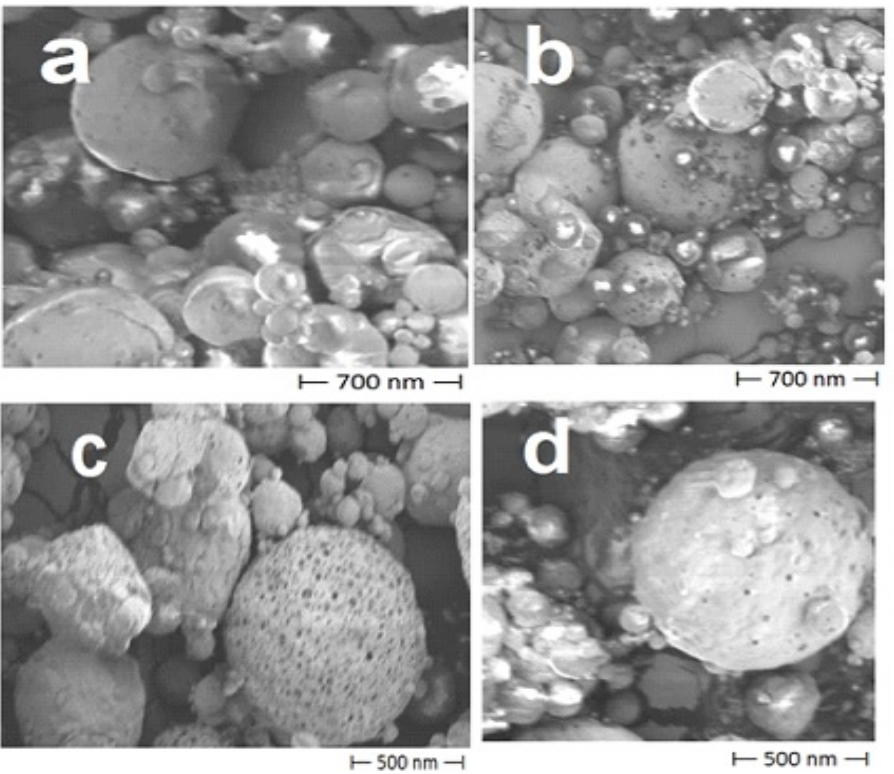

Figure 2: SEM micrographs of SLN's showing the shape of (a) EVG - SLN1, (b) EVG - SLN2, (c) EVG - SLN3 and (d) EVG - SLN4

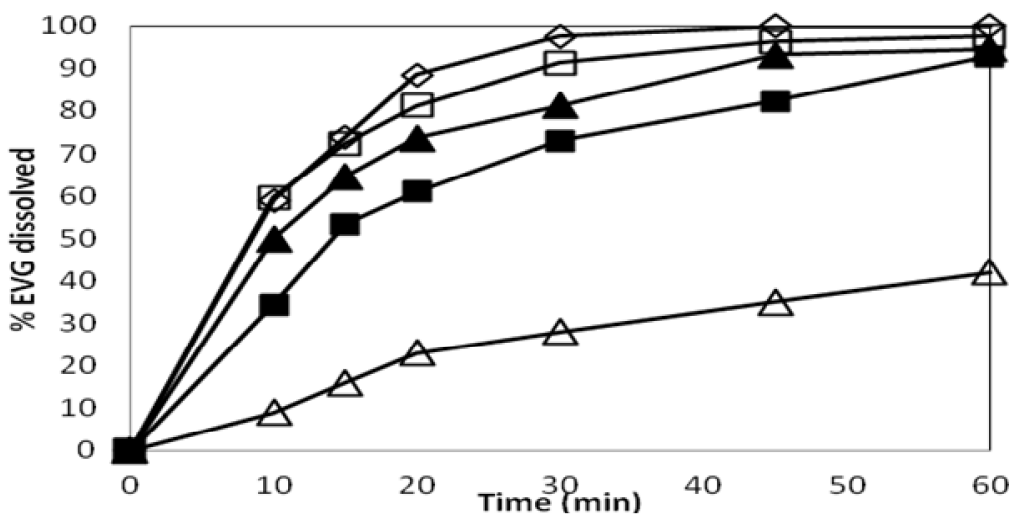

Figure 3: Dissolution profile of EVG - SLN systems compared to EVG, Note: $\Delta$ = EVG; $\mathbf{-}=\mathrm{EVG}-\mathrm{SLN1} ; \boldsymbol{\Delta}=$ EVG - SLN2; $\square=$ EVG - SLN3; $\diamond=E V G ~-~ S L N 4$ 
Coefficient of determination $\left(\mathrm{R}^{2}\right)$, scale parameter (a) and shape parameter (b) results for EVG-SLN'S are tabulated in Table 4.

Table 4 also presents the values of Dissolution efficiency (DE) which is the area under the dissolution curve between time point's $\mathrm{t} 1$ and $\mathrm{t} 2$ expressed as a percentage of the curve at maximum dissolution, $y 100$, over the same period and is expressed as in Eq 2.

$\mathrm{DE}=\left\{\left[\int_{\mathrm{t} 1}{ }^{2} \mathrm{y} \cdot \mathrm{dt}\right] /[\mathrm{y} 100(\mathrm{t} 2-\mathrm{t} 1)]\right\} \times 100$

DE values are related with the real amount of drug dissolved in the dissolution medium and thus, lead to a better extrapolative for in vivo performance [22]. From the table 4 it is observed that $D E$ increased as the lipid concentration increased.

Statistical assessment of the dissolution data was performed using analysis of variance (ANOVA-single factor). From ANOVA - single factor results of EVG- SLN's dissolution data, $F$ value of EVG - SLN1, EVG - SLN2, EVG - SLN3 and EVG - SLN4 is found to be 9.6, 28.5, 19.3 and 29.5 respectively. The F-value for all the SLN preparations was > Fcrit-value, i.e., 4.96. The p-values of EVG - SLN1, EVG - SLN2, EVG - SLN3 and EVG - SLN4 are found to be 0.0112, $0.0003,0.0013$ and 0.0003 respectively which are significantly smaller than 0.05 .

Based on the extremely low $p$-values and $F$ value $>$ Fcrit-value it can be deduced that there is a significant difference between the groups i.e. each of the EVG - SLN preparation significantly different in terms of percent dissolved at each time point. Further statistical assessment of the EVG and EVG SLN's dissolution data were performed using t-Test: Paired Two Sample for Means. The absolute $t$ Stat values for EVG SLN1, EVG - SLN2, EVG - SLN3 and EVG SLN4 were found to be $10.826,21.505,32.436$ and 21.072 respectively which are greater than $t$ Critical two - tail value 2.571. Hence, from these findings it was concluded that there is a difference in the drug release profiles of EVG and EVG SLN's.

\section{DISCUSSION}

The use of solvent injection method for the preparation of EVG loaded SLN's found to be advantageous in obtaining SLN with small, monodisperse nanoparticles with high Entrapment efficiency. The measurement of $Z$ potential allows the prediction of the storage stability of colloidal dispersion [16]. The $Z$ potential values did not change notably by increasing the lipid load and the obtained $Z$ potential values indicate the formation of stable suspension and there is no particle aggregation observed which is due to charged particles with high Z - potential due to electric repulsion $[17,18]$. In the present study, stable EVG - SLNs was prepared with $Z$ - potential values ranging between $-41.4 \pm 1.6$ to $-37.4 \pm 1.0$. When the cryoprotectants are added in different concentrations to improve the chemical and physical stability of SLN over an extended period of time during lyophilisation and from Z - potential values it was observed that EVG - SLN1and EVG - SLN2 were stable over entire concentration range but for EVG - SLN3 and EVG - SLN4 decline in the Z - potential values ranging from $29.5 \pm 0.7$ to $-8.60 \pm 3.6$ has been observed which may be due to the damage of surfactant film around the nanoparticle due to a freezing out effect and may also because of particle aggregation during the redispersion process $[19,20]$.

There was a tremendous increase in solubility and dissolution rate and this may be due to reduction in particle size resulting in increase in surface area and saturation solubility which is a key factor for improving the performance of poorly soluble drugs. In vitro release tests have confirmed that release velocity from SLNs is significantly faster and the release kinetics of the drug from EVG - SLN's is best described by Weibull mathematical model.

Table 4: Parameters of Weibull mathematical model and dissolution efficiency (DE) results for the dissolution data of SLN's

\begin{tabular}{lcccc}
\hline $\begin{array}{l}\text { Formulation } \\
\text { Name }\end{array}$ & $\begin{array}{c}\text { Coefficient of } \\
\left.\text { determination } \mathbf{( R}^{2}\right)\end{array}$ & $\begin{array}{c}\text { Shape parameter } \\
(\mathbf{b})\end{array}$ & $\begin{array}{c}\text { Scale } \\
\text { parameter (a) }\end{array}$ & $\begin{array}{c}\text { Dissolution } \\
\text { efficiency (DE, \%) }\end{array}$ \\
\hline EVG - SLN1 & 0.9805 & 0.95 & 0.95 & 63.6 \\
EVG - SLN2 & 0.9883 & 0.82 & 9.03 & 72.9 \\
EVG - SLN3 & 0.9972 & 0.82 & 7.16 & 78.8 \\
EVG - SLN4 & 0.9262 & 2.15 & 219.55 & 82.9 \\
\hline
\end{tabular}


Weibull equation expresses accumulated fraction of drug $(\mathrm{m})$, in solution at time at time (t) and is given by following expression:

$m=1-\exp \left\{\left[-(t-T i)^{b}\right] / a\right\}$

In this equation (3), ' $a$ ' is the scale parameter which defines the time scale of the process. ' $b$ ' is the shape parameter which characterizes the curve as either sigmoid $(b=1), S$ - shaped with upward curvature followed by a turning point $(b>$ 1) or parabolic, with a higher initial slope and after that consistent with the exponential $(b<1)$. ' $\mathrm{Ti}$ ' is the location parameter which represents the lag time before the onset of the dissolution and in most cases it will be zero [21].

The use of SLNs opens up new perspectives for the formulation of poorly soluble drugs. The poor aqueous solubility of EVG also makes it difficult to prepare parenteral formulations which can be overcome using SLN concept.

\section{CONCLUSION}

This study demonstrates that EVG loaded SLN's were successfully prepared and characterized. Thus, solvent injection technique seems a suitable method for the preparation of nanosuspension of poorly water-soluble drugs, such as elvitegravir, for enhancement of dissolution rate.

\section{ACKNOWLEDGEMENT}

The authors wish to thank Satyavani Battula (BITS - Hyderabad, India) for participating assisting with particle measurement.

\section{REFERENCES}

1. Mehnert W, Mäder K. Solid lipid nanoparticles: Production, characterization and applications. Adv Drug Deliv Rev 2001; 47: 165-196.

2. MuËller RH, MaËder K, Gohla S. Solid lipid nanoparticles (s/n) for controlled drug delivery-a review of the state of the art. Eur J Pharm Biopharm 2000; 50: 161-177.

3. Müller RH, Maaßen S, Weyhers H, Specht F, Lucks JS. Cytotoxicity of magnetite-loaded polylactide, polylactide/glycolide particles and solid lipid nanoparticles. Int J Pharm 1996; 138: 85-94.

4. Yang S, Zhu J, Lu Y, Liang, B, Yang C. Body distribution of camptothecin solid lipid nanoparticles after oral administration. Pharmaceut Res 1999; 16: 751-757.

5. Kaur IP, Bhandari R, Bhandari S, Kakkar, V. Potential of solid lipid nanoparticles in brain targeting. J Control Release 2008; 127: 97-109.
6. Mukherjee S, Ray S, Thakur RS. Solid lipid nanoparticles: $A$ modern formulation approach in drug delivery system. Indian J Pharm Sci 2009; 71: 349.

7. Schwarz C, Mehnert W, Lucks JS, Müller RH. Solid lipid nanoparticles (s/n) for controlled drug delivery. I. Production, characterization and sterilization. J Controlled Release 1994; 30: 83-96.

8. Wissing $S A$, Kayser $O$, Müller $R H$. Solid lipid nanoparticles for parenteral drug delivery. Adv Drug Deliver Rev 2004; 56: 1257-1272.

9. Schubert M, Müller-Goymann C. Solvent injection as a new approach for manufacturing lipid nanoparticlesevaluation of the method and process parameters. Eur J Pharm Biopharm 2003; 55: 125-131.

10. Mishra D, Mishra H, Mishra PK, Nahar M, Dubey V, Jain NK. Evaluation of solid lipid nanoparticles as carriers for delivery of hepatitis $b$ surface antigen for vaccination using subcutaneous route. I Pharm Pharm Sci 2010; 13: 495-509.

11. Kaushik MM, Shukla TP, Upadhyay N, Mathur A, Cherian $B P$. Formulation development and evaluation of solid lipid nanoparticles of aceclofenac using solvent injection method. Journal of Drug Delivery and Therapeutics 2012; 2(4), 97-100.

12. Shimura K, Kodama E, Sakagami Y, Matsuzaki $Y$, Watanabe W, Yamataka K, Watanabe y, Matsuoka $M$. Broad antiretroviral activity and resistance profile of the novel human immunodeficiency virus integrase inhibitor elvitegravir (jtk-303/gs-9137). J Virol 2008; 82: 764-774.

13. DeJesus E, Berger D, Markowitz M, Cohen C, Hawkins $T$, Ruane $P$, Elion $R$, et al. Antiviral activity, pharmacokinetics, and dose response of the hiv-1 integrase inhibitor gs-9137 (jtk-303) in treatmentnaive and treatment-experienced patients. J Acq Immun Def Synd 2006; 43: 1-5.

14. Ramanathan $S$, Mathias $A A$, German $P$, Kearney $B P$. Clinical pharmacokinetic and pharmacodynamic profile of the hiv integrase inhibitor elvitegravir. Clin Pharmacokinet 2011; 50: 229-244.

15. Quintanar-Guerrero D, Allémann E, Doelker E, Fessi H. A mechanistic study of the formation of polymer nanoparticles by the emulsification-diffusion technique. Colloid Polym Sci 1997; 275; 640-647.

16. Komatsu H, Kitajima A, Okada S. Pharmaceutical characterization of commercially available intravenous fat emulsions: Estimation of average particle size, size distribution and surface potential using photon correlation spectroscopy. Chem Pharm Bulletin 1995; 43: 1412-1415.

17. Levy MY, Schutze W, Fuhrer C, Benita S. Characterization of diazepam submicron emulsion interface: Role of oleic acid. J Microencapsul 1994; 11: 79-92.

18. Takamura A, Ishii $F$, Noro SI, Tanifuji M, Nakajima $S$. Study of intravenous hyperalimentation: Effect of 
selected amino acids on the stability of intravenous fat emulsions. J Pharm Sci 1984; 73: 91-94.

19. Mehnert W, Mäder K. Solid lipid nanoparticles: Production, characterization and applications. Adv Drug Deliv Rev 2001; 47: 165-196.
20. Zimmermann E, Müller RH, Mäder $K$. Influence of different parameters on reconstitution of lyophilized sln. Int J Pharm 2000; 196: 211-213.

21. Langenbucher $F$. Letters to the editor: Linearization of dissolution rate curves by the weibull distribution. $J$ Pharm Pharmacol 1972; 24: 979-981. 